\title{
TECTA mutations in Japanese with mid-frequency hearing loss affected by zona pellucida domain protein secretion
}

\author{
Hideaki Moteki ${ }^{1}$, Shin-ya Nishio ${ }^{1}$, Shigenari Hashimoto ${ }^{1}$, Yutaka Takumi ${ }^{1}$, Satoshi Iwasaki ${ }^{2}$, \\ Norihito Takeichi ${ }^{3}$, Satoshi Fukuda ${ }^{3}$ and Shin-ichi Usami ${ }^{1}$
}

TECTA gene encodes $\alpha$-tectorin, the major component of noncollagenous glycoprotein of the tectorial membrane, and has a role in intracochlear sound transmission. The TECTA mutations are one of the most frequent causes of autosomal dominant (AD) hearing loss and genotype-phenotype correlations are associated with mutations of TECTA in exons according to $\alpha$-tectorin domains. In this study, we investigated the prevalence of hearing loss caused by TECTA mutations in Japanese AD hearing loss families, and confirmed genotype-phenotype correlation, as well as the intracellular localization of missense mutations in the $\alpha$-tectorin domain. TECTA mutations were detected in $2.9 \%$ (4/139) of our Japanese AD hearing loss families, with the prevalence in moderate hearing loss being $7.7 \%(4 / 52)$, and all patients showed typical genotype-phenotype correlations as previously described. The present in vitro study showed differences of localization patterns between wild type and mutants, and suggested that each missense mutation may lead to a lack of assembly of secretion, and may reduce the incorporation of $\alpha$-tectorin into the tectorial membrane.

Journal of Human Genetics (2012) 57, 587-592; doi:10.1038/jhg.2012.73; published online 21 June 2012

Keywords: autosomal dominant hearing loss; genotype-phenotype correlations; mid-frequency hearing loss; TECTA; zona pellucida domain

\section{INTRODUCTION}

Hearing loss affects about 1 in 500-1000 newborns in developed countries and genetic causes account for at least $50 \%$ of all childhood nonsyndromic sensory neural hearing loss (SNHL). ${ }^{1}$ Most of these cases are affected with severe and congenital prelingual deafness and autosomal recessive inheritance represented by GJB2 gene mutations predominates $(80 \%)$ over autosomal dominant (AD) $(20 \%){ }^{2}$ Mild to moderate SNHL and/or late-onset SNHL, presenting with $\mathrm{AD}$ inheritance pedigree pattern, is commonly supposed to be of genetic causes. $\mathrm{AD}$ nonsyndromic hearing loss (ADNSHL) is represented by heterogeneity of genetic and clinical features, as 60 loci have been mapped, 24 genes have been cloned and correlation with audiological features have been reported (Van Camp and Smith, Hereditary Hearing Loss Homepage. WorldWide Web URL: http://hereditaryhearingloss.org). These types of SNHL can be characterized by age of onset, progression and pattern of audiogram.

As one cause of ADNSHL, TECTA mutations have been found in various types of hearing loss, age of onset, progression and frequency involvement in various populations. ${ }^{3-10}$ This gene encodes $\alpha$-tectorin, the major component of noncollagenous glycoprotein of the tectorial membrane that consists of an extracellular matrix overlying the organ of corti, contacting the outer cochlear hair cells, and having a role in intracochlear sound transmission. ${ }^{11}$ The $\alpha$-tectorin is composed of three distinct modules: the entactin G1 domain, the zonadhesin (ZA) domain with von Willebrand factor type $\mathrm{D}$ repeats and the zona pellucida (ZP) domain. ${ }^{11}$ No nonsense mutations of TECTA have been reported in $\mathrm{AD}$ hearing loss. Missense mutations affecting the $\mathrm{ZP}$ domain are associated with mid-frequency hearing impairment, whereas mutations in the ZA domain are associated with hearing impairment primarily affecting the high frequencies. ${ }^{12}$ Phenotypes of hearing loss can range from mild to severe and have pre or postlingual onset. $^{8}$

In this study, (1) we examined the prevalence of hearing loss caused by TECTA mutations in Japanese ADSHNL and confirmed genotypephenotype correlation, and (2) examined the impact of three missense mutations in the ZP domain on the cellular distribution of $\alpha$-tectorin, known to cause mid-frequency hearing impairment. Many deafnesscausing TECTA mutations have been reported, but the molecular mechanisms are unclear. To investigate the biological function of missense mutations in the $\mathrm{ZP}$ domain that were reported as causing ADSHNL GFP fusion proteins were generated and the effects of

${ }^{1}$ Department of Otorhinolaryngology, Shinshu University School of Medicine, Matsumoto, Japan; ${ }^{2}$ Department of Hearing Implant Sciences, Shinshu University School of Medicine, Matsumoto, Japan and 3 Department of Otolaryngology Head and Neck Surgery, Hokkaido University School of Medicine, Sapporo, Japan 
corresponding mutations on secretion patterns of the ZP domain of $\alpha$-tectorin were examined.

\section{MATERIALS AND METHODS}

\section{Subjects}

A total of 139 Japanese AD (with two or more generations affected) sensorineural hearing loss families were screened for mutations in the TECTA gene. All probands were from independent families and none had any other associated neurological signs, visual dysfunction or diabetes mellitus. Hearing level was classified by a pure-tone audiometry average over 500, 1000, 2000 and $4000 \mathrm{~Hz}$ in the better hearing ears as follows: normal hearing, $<20 \mathrm{~dB}$; mild hearing loss, 21-40 dB; moderate hearing loss, 41-70 dB; severe hearing loss, 71-95 dB; and profound hearing loss, >95 dB (GENDEAF, 2004). Of the 139 probands, 4 (3\%) had normal hearing (only limited frequencies involved), $40(29 \%)$ had mild hearing loss, $52(37 \%)$ had moderate hearing loss, 23 (17\%) had severe hearing loss and $12(9 \%)$ had profound hearing loss Information on pure-tone audiometry was not available for eight $(6 \%)$ of these subjects. The mean age at their participation (not onset of hearing loss) of the subjects were; normal hearing, $14.0 \pm 10.6$ years; mild hearing loss, $21.1 \pm 15.5$ years; moderate hearing loss, $25.3 \pm 19.0$ years; severe hearing loss, $32.1 \pm 25.5$ years; and profound hearing loss, $27.5 \pm 19.2$ years.

All subjects gave prior informed written consent for participation in this study and the Ethical Committee of Shinshu University approved the study.

\section{Mutation analysis}

All 23 exons and flanking intronic sequences of the TECTA gene were amplified by PCR. Primers were designed to flank all of the exon-intron boundaries by use of the Primer3 web-based server (http://frodo.wi.mit.edu/). Each genomic DNA sample (40 ng) was amplified, using Ex-Taq polymerase (Takara, Otsu, Japan), for $5 \mathrm{~min}$ at $95^{\circ} \mathrm{C}$, followed by 37 three-step cycles of $95^{\circ} \mathrm{C}$ for $30 \mathrm{~s}, 56-63{ }^{\circ} \mathrm{C}$ for $30 \mathrm{~s}$ and $72^{\circ} \mathrm{C}$ for $1.5 \mathrm{~min}$, with a final extension at $72^{\circ} \mathrm{C}$ for $10 \mathrm{~min}$, ending with a holding period at $4{ }^{\circ} \mathrm{C}$ in a Perkin-Elmer thermal cycler (Perkin-Elmer, Wellesley, MA, USA). The PCR products varied in size at about $200-700 \mathrm{bp}$, and they were treated with $0.1 \mu \mathrm{l}$ exonuclease I (Amersham) and $1 \mu \mathrm{l}$ shrimp alkaline phosphatase (Amersham, Princeton, NJ, USA) and by incubation at $37^{\circ} \mathrm{C}$ for $30 \mathrm{~min}$, and inactivation at $80^{\circ} \mathrm{C}$ for $15 \mathrm{~min}$. After the products were purified, we performed standard cycle sequencing reaction with ABI Big Dye terminators in an ABI 3100 autosequencer (Applied Biosystems, Foster City, CA, USA).

\section{cDNA products ZP domain expression plasmids}

Full-length cDNAs of the ZP domains of $\alpha$-tectorin genes were cloned by conventional PCR from the human fetal brain cDNA library (Invitogen, Carlsbad, CA, USA). Two pairs of primers for the entire coding regions of $\mathrm{ZP}$ domain including transmembrane domain of $\alpha$-tectorin were used. PCR steps were denaturing at $94{ }^{\circ} \mathrm{C}$ for $2 \mathrm{~min}$, followed by 30 cycles of $94{ }^{\circ} \mathrm{C}$ for $30 \mathrm{~s}$, $53^{\circ} \mathrm{C}$ for $30 \mathrm{~s}$ and $72^{\circ} \mathrm{C}$ for $1 \mathrm{~min}$, and then processing with a final extension at $72{ }^{\circ} \mathrm{C}$ for $5 \mathrm{~min}$. After amplification, expected sizes of PCR products were confirmed on $2 \%$ agarose gel, and the bands were visualized by ethidium bromide upon exposure to an ultraviolet transilluminator.

Produced cDNAs were digested with BamHI/EcoRI and cloned into the BamHI/EcoRI site of pEGFP-C2 vecter (Clontech, Palo Alto, CA, USA). Ligation reactants were transformed into Escherichia coli DH5a. A QIAprep spin miniprep kit (Qiagen, Valencia, CA, USA) was used for purification of plasmid DNA according to the manufacturer's protocol.

\section{Site-directed gene mutagenesis}

Gene mutagenesis of the ZP domain cDNA was performed by mega primer PCR. ${ }^{13}$ The mutatagenesis primers for 5509TG, 5876A $>\mathrm{G}$ and $6063 \mathrm{G}>\mathrm{A}$, which were previously reported from Spain, ${ }^{4}$ Austria ${ }^{14}$ and Japan, ${ }^{5}$ respectively, were designed. The following reverse primers were used to produce the mutations for initial PCR reaction: 5509TG CCCCTCGATGCCGGTGCCC TGTCTGTCA, 5876AG TCCAGAGTGTGTTTTTACACATGATATG and 6063GA ACCGAGCTGGAAGAACTTGCACTTAGAT. Initial PCR reactions
$(20 \mu \mathrm{l})$ were prepared containing $0.1 \mu \mathrm{g}$ of template DNA, $0.4 \mu \mathrm{M}$ of mutation primer, $0.4 \mu \mathrm{M}$ of ZP-EcoRI primer, $0.1 \mathrm{U}$ of KOD pulse (TOYOBO, Osaka, Japan) and KOD buffer, $2.0 \mu \mathrm{M} \mathrm{MgSO}_{4}$ and $0.8 \mu \mathrm{M} \mathrm{dNTP}$ These PCR reactions were denatured at $94^{\circ} \mathrm{C}$ for $2 \mathrm{~min}$, followed by 30 cycles of $94^{\circ} \mathrm{C}$ for $30 \mathrm{~s}, 55^{\circ} \mathrm{C}$ for $30 \mathrm{~s}$ and $72^{\circ} \mathrm{C}$ for $1 \mathrm{~min}$, and then processed with a final extension at $72^{\circ} \mathrm{C}$ for $5 \mathrm{~min}$. The subsequent mega primer reactions were prepared containing initial PCR products, which were diluted 50-fold each, $0.4 \mu \mathrm{M}$ of ZP-BamHI R primer, 2.0 U of Takara Ex-taq (Takara) and Ex-taq buffer $(10 \times)$. These PCR products were inserted into a pEGFP-C2 vector with the same techniques as above. The sequences of all three cDNA constructs were confirmed by DNA sequencing using an ABI 3100 autosequencer.

\section{Transfection and confocal microscopy}

COS-7 cells grown in Dulbecco's modified Eagle medium (Mediatech, Herndon, VA, USA) supplemented with $10 \%$ fetal calf serum (Moregate, Bulimba, QLD, Australia) were transiently transfected with the indicated plasmids, using Lipofectamine 2000 (Invitrogen) as described by the manufacturer. At $24 \mathrm{~h}$ after the transfection, cells were washed twice with phosphatebuffered saline. Cover slips were mounted onto glass slides and visualized under a Leica confocal microscope TCS SP2 AOBS (Leica Microsystems, Wetzlar, Germany).

\section{RESULTS}

\section{Mutation screening of the TECTA gene}

Direct DNA sequencing identified four pathogenic mutation alleles from $\mathrm{AD}$ families, including one family in which two mutations were found in one allele. Among those, the family with the c.6063G $>$ A (p.R2021H) mutation was previously reported by Iwasaki et al. ${ }^{5}$ Including those results, TECTA mutations were detected in $2.9 \%$ (4/139) of Japanese ADNSHL families, and the prevalence in moderate hearing loss was $7.7 \%(4 / 52)$.

The family F818 pedigree consisted of three generations and included nine affected members (four males and five females), four of whom participated in this study (Figure 1). This family had a p.R1773X (c.5318C > T) mutation affecting the ZA domain in exon 16 , and had slowly progressive high frequency hearing loss. Segregation with hearing loss was confirmed in all cases for which DNA samples were available and none of the mutations were detected in controls.

The family F237 pedigree consisted of three generations and included five affected members (three males and two females), four of whom participated in this study (Figure 2). They demonstrated bilateral mild to moderate symmetric sensorineural hearing loss and showed a U-shaped audiogram, affected in the mid frequencies. Vestibular disorder symptoms were not observed, and inner ear abnormalities were not found with CT scans. Two missense mutations in one allele, p. [H1400W; T1866M] (c. [4198C > T; 5597C > T]), were detected in $\alpha$-tectorin in this family. The mutation $\mathrm{H} 1400 \mathrm{~W}$ in exon 12 was in the ZA domain of $\alpha$-tectorin, whereas T1866M was in the $\mathrm{ZP}$ domain.

The family $\mathrm{F} 652$ pedigree consisted of four generations and included 16 affected members (Figure 3). I1997T (c.5990T >C) mutations were detected in $\alpha$-tectorin in this family. The mutation in exon 19 located in the ZP domain of $\alpha$-tectorin. This missense mutation appeared in heterozygosity and was shown to segregate almost completely with the affected status in this family. The audiograms were symmetric and often showed a $U$ shape, which indicates that predominantly the mid frequencies are affected. But, one member, a 11-year-old girl (Figure 3a(III-6)), although bearing the responsible mutation, had a normal audiogram and no demonstrable hearing loss. 
a

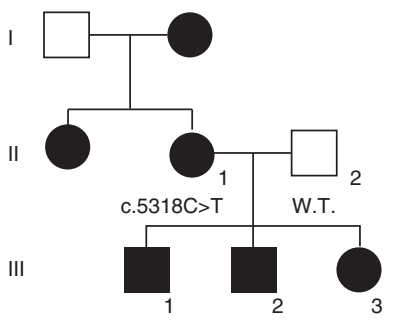

c. $5318 \mathrm{C}>\mathrm{T} \quad$ c. $5318 \mathrm{C}>\mathrm{T} \quad$ c. $5318 \mathrm{C}>\mathrm{T}$
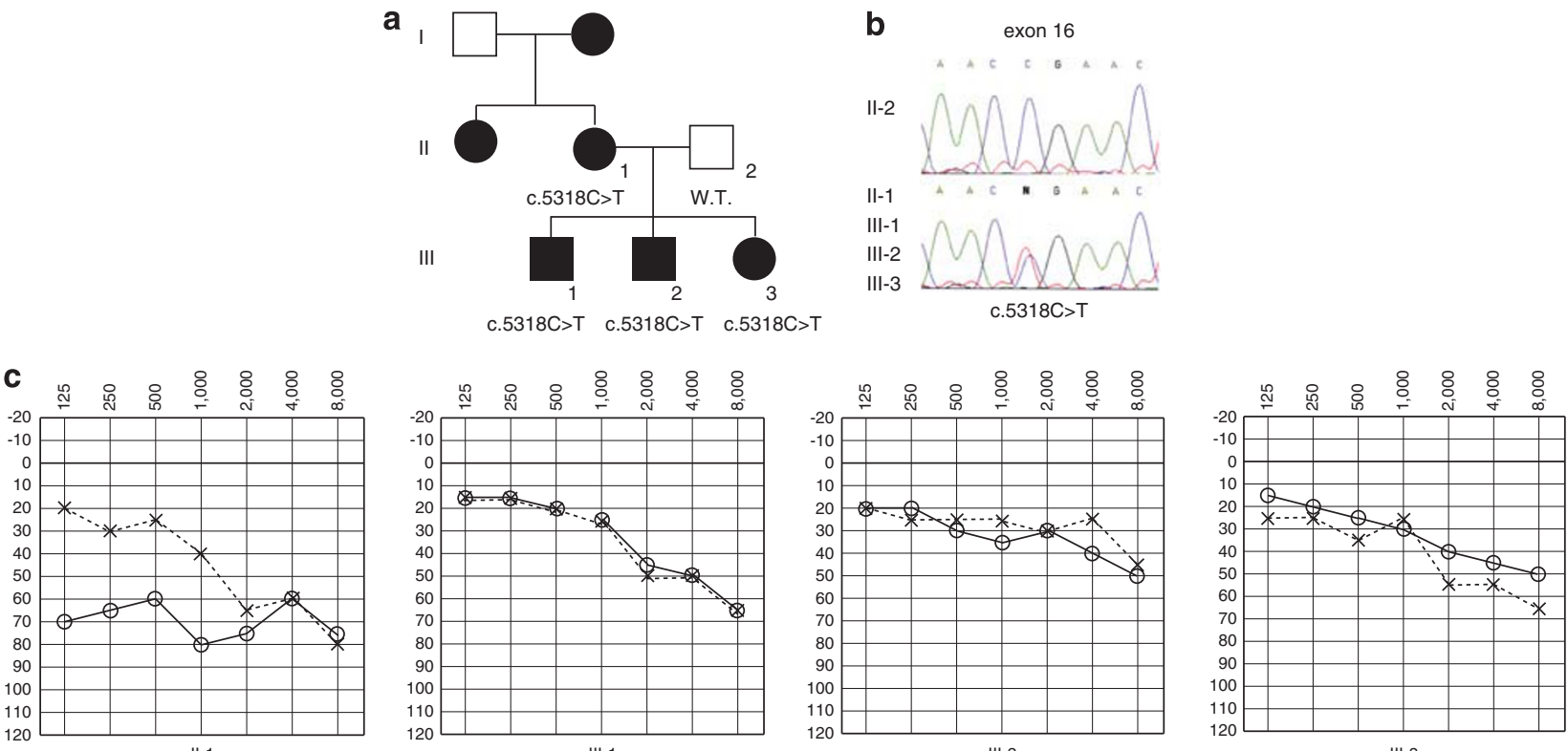

III-1

III-2

III-3

Figure 1 (a) Pedigree of the family F818 and audiograms of four different patients. Black and white symbols indicate the affected and the unaffected subjects, respectively. (b) Electropherograms for unaffected (wt) and affected family members showing the heterozygous c.5318C $>$ T mutation of TECTA co-segregating with hearing loss in this family. (c) Audiograms of four different affected patients show high frequency hearing loss. Patient II-1 suffered decreasing hearing level in the right ear with cholesteatoma and postoperative change.

a

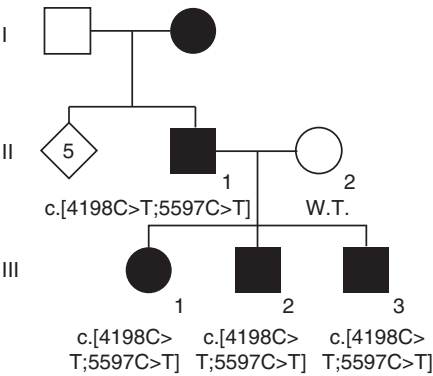

b

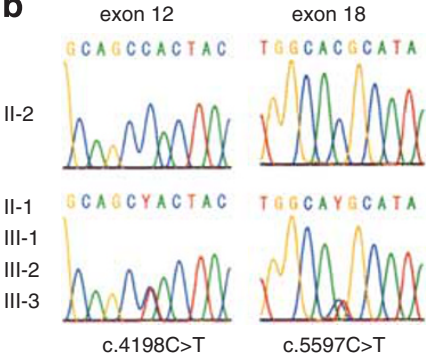

C

H.sapiens KSDCSHYCVE...GNIVQSNGTHIMYKNT

P.troglodytes KSDCSHYCVE... GNIVQSNGTHIMYKNT C.lupus KSECNHYCVE. . . GNIVQSNGTHIMYKNT B.taurus KSDCNHYCVE...GNIVQSNGTHIMYKNT M.musculus KSDCNHYCVE... GNIVQSNGTHIMYKNT R.norvegicus KSDCNHYCVE... GNVVQSNGTHIMYKNT G.gallus KSDCGHYCVE...GNLVQSNSTHIVYKNT D.rerio QRDCNOYCVE...GSIVQSNGTHIMYKNT
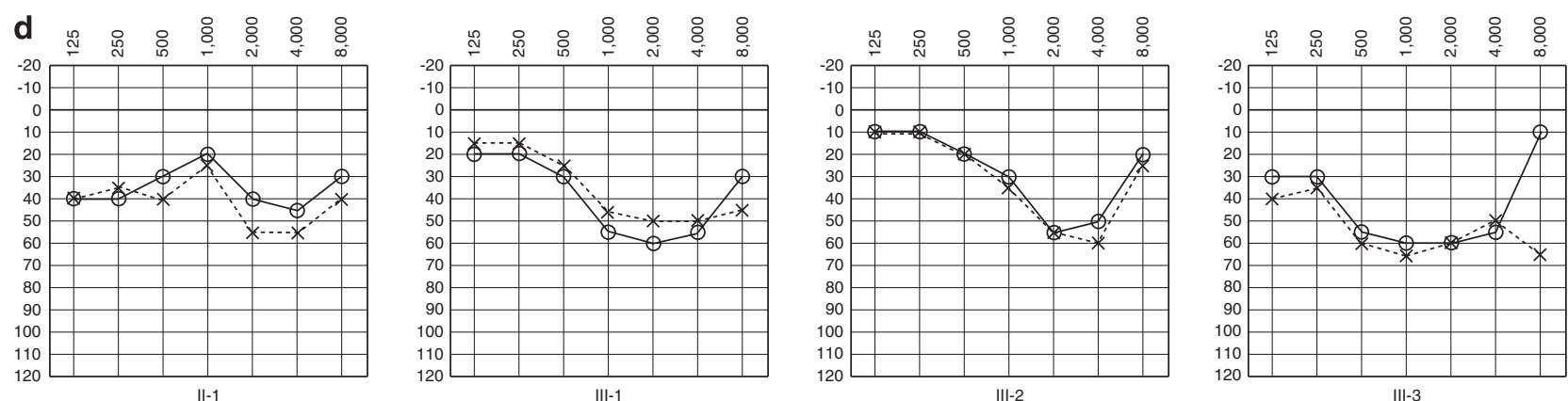

Figure 2 (a) Pedigree of the family F237 and audiograms of four different patients. The marks and symbols are as described in Figure 1. (b) Electropherograms for unaffected (wt) and affected family members showing the heterozygous c.4198C > T and c.5597C > T mutations of TECTA. (c) Multiple amino-acid alignment of proteins homologous to the alfa-tectorin ZP domain containing these mutated positions. Amino-acid residues that are identical among all of the homologs are enclosed. (d) Audiograms of four different affected patients showing deterioration in mid-frequency as a U-shaped audiogram.

\section{Localization of ZP domain mutants}

The inherent fluorescence of GFP determined the intracellular localization of the recombinant fusion proteins (Figure 4). Transfected GFP-ZP domains of $\alpha$-tectorin wt (wild type) were found to be localized as labeled puncta, which may be secreted along the plasma membrane. In contrast, GFP-ZP domains of $\alpha$-tectorin mutants, (GFP-ZP mut) C1837G, Y1870C and R2021H, were not recognized at the plasma membrane but were retained within the cytoplasm where they formed vesicles.

\section{DISCUSSION}

We have identified four independent AD families associated with four different TECTA mutations. Before this study, one Japanese family with $6063 \mathrm{G}>\mathrm{A}(\mathrm{R} 2021 \mathrm{H})$ mutation had been reported. ${ }^{5}$ Including 


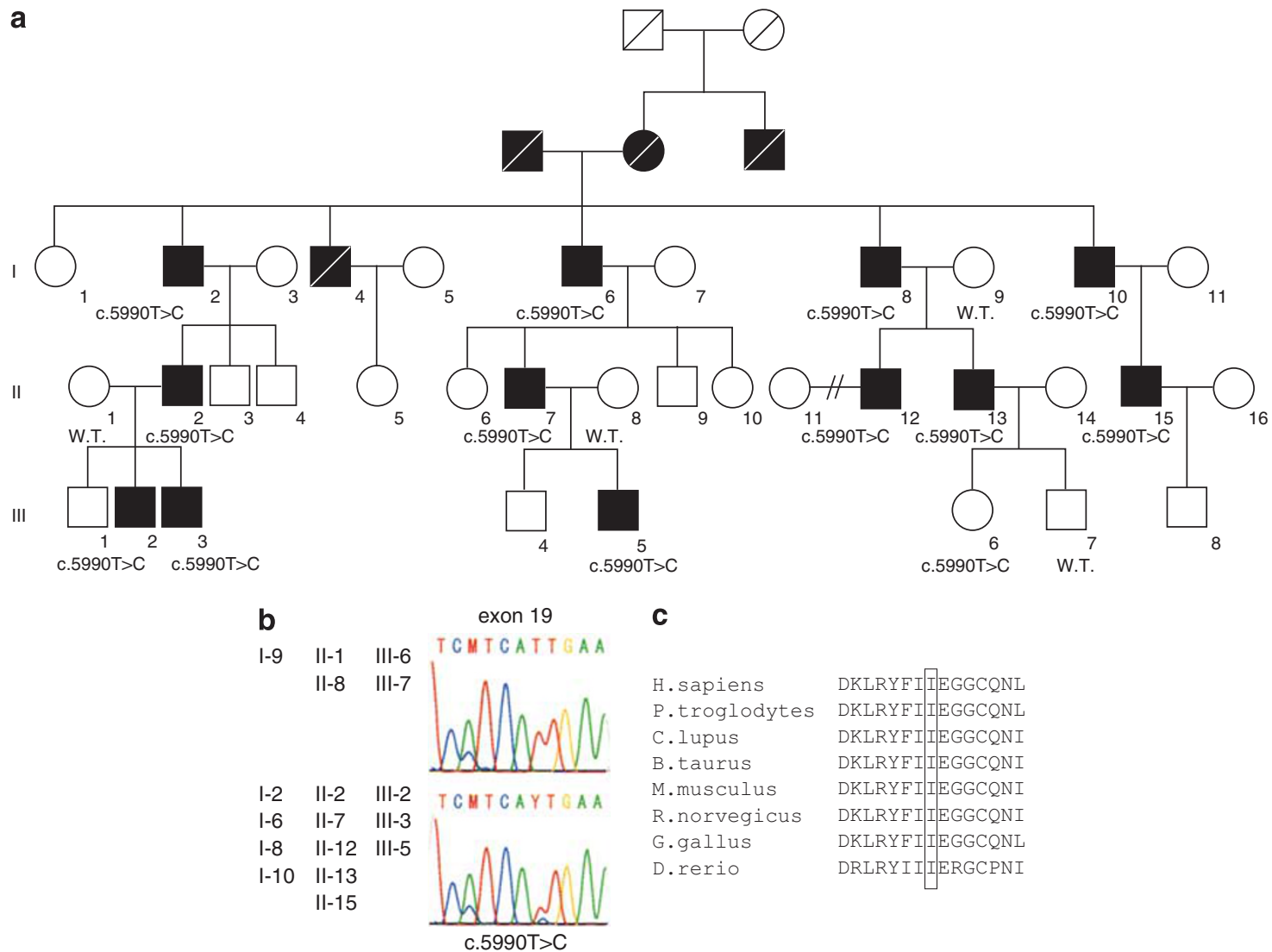

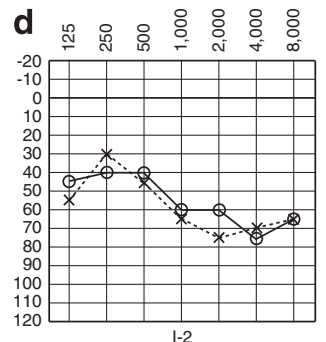
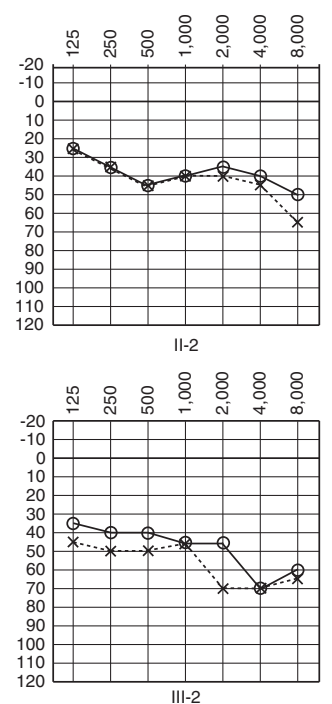

电 \& \& \& : \&

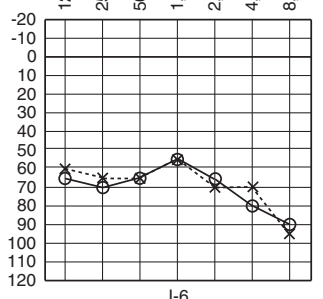

虬

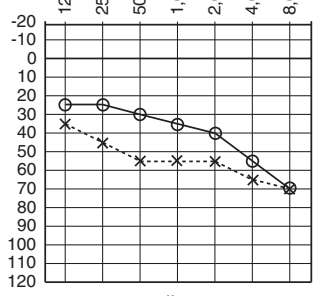

11-7

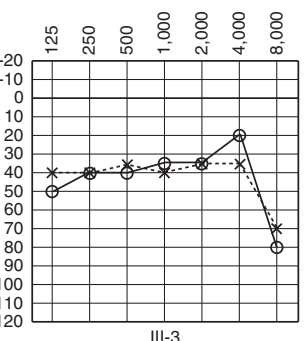

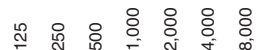

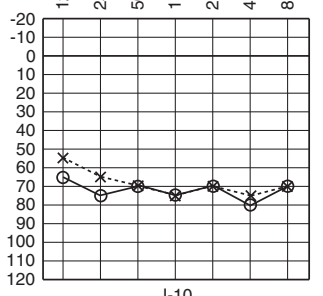

虫

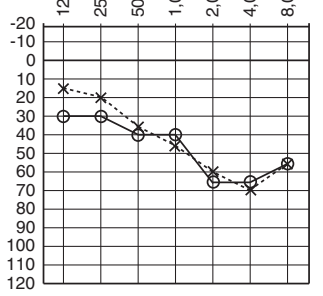

॥-12

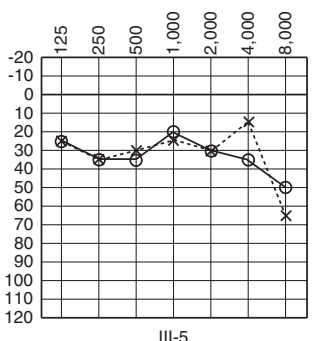

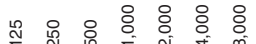

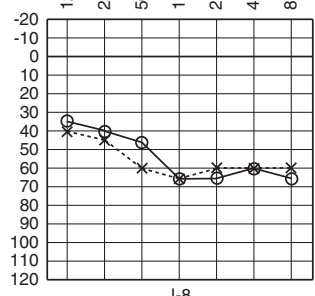

品

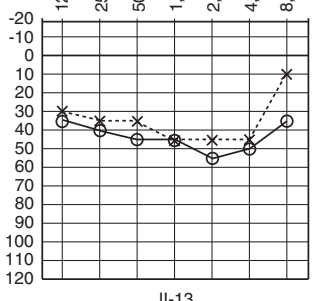

II-13

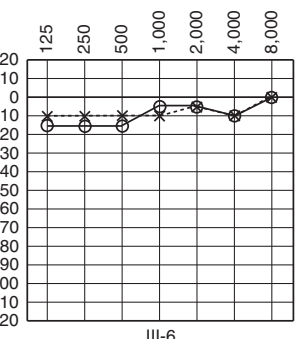

怘通

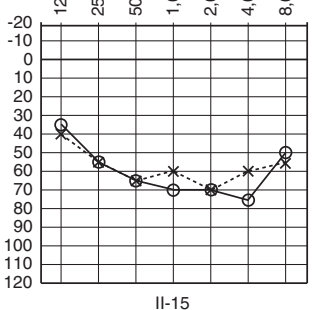

Figure 3 (a) Pedigree of the family F652, (b) electropherograms for unaffected (wt) and affected family members showing the heterozygous c.5990T >C mutation of TECTA. (c) C.5990T >C is predicted to substitute isoleucine for threonine acid at amino acid position 1997. Multiple amino-acid alignment of protein homologs was conserved. (d) Audiograms of affected patients were flat to U-shaped, and there was a tendency to decreased hearing level associated with age. 


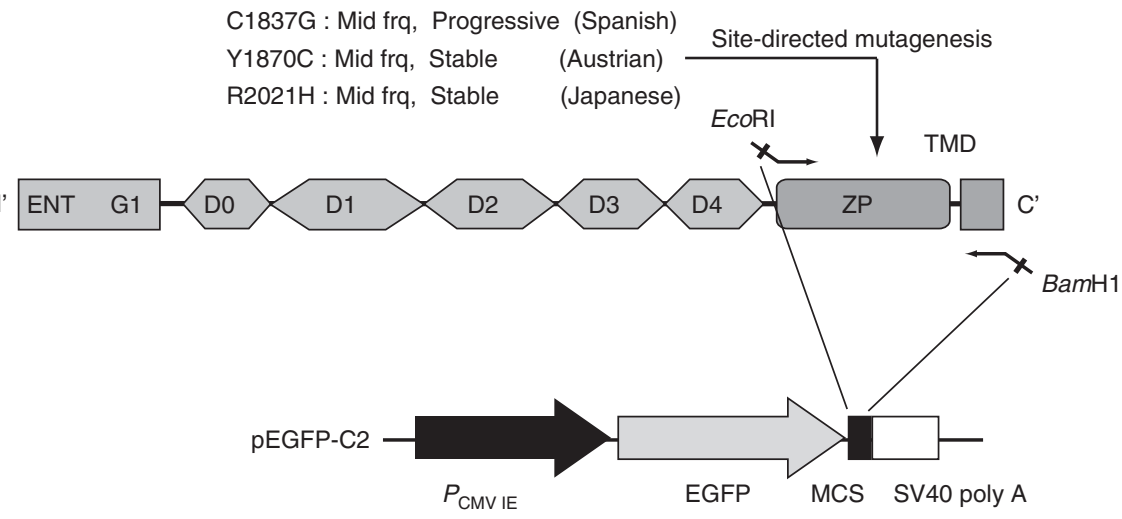

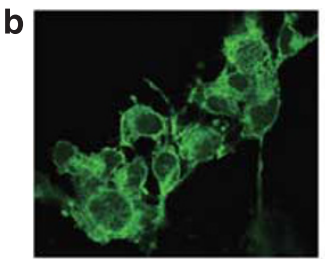

WT

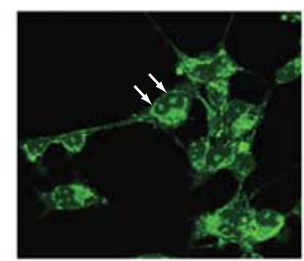

C1837G

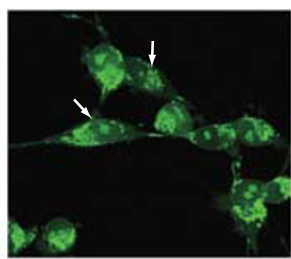

$\mathrm{R} 2021 \mathrm{H}$

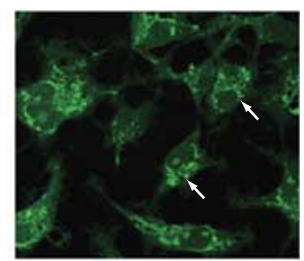

Y1870C

Figure 4 (a) Domain structure of the human alfa-tectorin protein. Three mutants in ZP domain protein including Transmembrane domain were generated by site-directed mutagenesis. Resulting CDNAs digested with EcoRI/ BamHI and cloned into the site of pEGFP-C2 plasmid are shown below. (b) Protein expression in COS-7 cells transfected with GFP-ZP wt showing a characteristic puncta along the plasma membrane. In contrast, GFP-ZP mutants (mut) C1837G, R2021H and Y1870C, which were associated with mid-frequency hearing loss phenotypes, were not recognized at the plasma membrane but were retained within the cytoplasm as white arrows indicated.

that family, prevalence of ADNSHL with TECTA mutation was $2.9 \%$ (4/139 families), which may be a relatively high incidence. Hildebrand et al. ${ }^{8}$ reported that its prevalence was about $4 \%$ in Spanish ADNSHL families (17/374 families). In our results, when limited to moderate hearing loss patients there was a higher rate of detection $(7.7 \% ; 4 / 52$ families).

In this study, all patients showed typical genotype-phenotype correlations of SNHL with TECTA mutation as previously described..$^{8,12,15}$ The family $\mathrm{F} 818$ in which the R1773X $(c .5318 \mathrm{C}>\mathrm{T})$ mutation in the $\mathrm{ZA}$ domain was detected, showed high frequency hearing loss that was slowly progressive. The affected proband (Figure 1) noticed bilateral hearing impairment when she was around age 20, and her right hearing level was worse than the left because of cholesteatoma in her right ear. Hearing impairment was detected in her sons in school health checks but they had never suffered vertigo and no inner ear abnormality was seen in CT scans.

In the family F237, two missense mutations, H1400W (c.4198C $>$ T) and T1866M (c.5597C $>$ T), were detected in $\alpha$ tectorin. The mutation H1400W in exon 12 was in the ZA domain of $\alpha$-tectorin, whereas T1866M was in the ZP domain. Both aminoacid residues were conserved among another species. It had been suggested in a previous report that TECTA-affected mid-frequency hearing impairment appeared to be related to the position of the mutations in the ZP domain of $\alpha$-tectorin. Considering the phenotype and position of the mutation, $T 1866 \mathrm{M}$ was likely to be causative for hearing impairment in this family. The influence of the nucleotide change of c.4198C $>\mathrm{T}$ on apparent effect of splicing of the TECTA mRNA cannot be predicted. However, because this change was not present in the controls, it cannot be ruled out that it has an effect on the phenotype of these patients or it may even act synergistically with the $\mathrm{T} 1866 \mathrm{M}$ (c.5597C $>\mathrm{T}$ ) mutation. The similar results with two changes in one family were reported by Plantinga ${ }^{12}$ in 2006 . The T1866M mutation that we detected in this study was previously reported in one family each in Korea, Spain and the USA.,8 Hildebrand reported that the Spanish and American cases do not suggest a founder effect for this mutation. ${ }^{8}$ Therefore, this T1866M mutation, now known to be existent in four independent families from four different countries, is suggested to be a possible mutational site hot spot.

In the family F652, we detected a novel mutation, I1997T (c.5990T >C), in exon 19 located in the ZP domain of $\alpha$-tectorin. The audiograms of affected patients indicated U-shaped midfrequency hearing loss, associated with a ZP domain mutation previously reported. Regarding progression of hearing loss, these audiograms showed that the thresholds depend on age among these generations. Accordingly, this mutation would lead to slowly progressive mid-frequency hearing loss. Interestingly, an affected female (Figure $3 \mathrm{a}(\mathrm{III}-6)$ ) exhibited normal hearing at the age of 12 . The other affected male members had been diagnosed with hearing loss between the ages of 10 and 15. Pfister had reported that there was gender difference in the severity of hearing loss in affected family members bearing the same TECTA mutations, with males being significantly more affected than females. ${ }^{16}$ Therefore, there is a need for more detailed audiologic analysis and follow-up in the other families to see whether they also show the same phenomenon in hearing impairment.

The present study further investigated whether the molecular mechanisms of hearing loss associated with TECTA mutations could be explained by protein expression. In contrast to COS-7 cells transfected with GFP-ZP wt, which were found to be localized in punctate spots along the plasma membrane (Figure $4 \mathrm{~b}$ ), the localization of GFP-ZP mutation proteins were not seen on the cellular membrane but mainly aggregated in the cytoplasm (Figure $4 \mathrm{~b}$ ). These mutations were located in the ZP domain of $\alpha$-tectorin, this domain is responsible for secretion and polymerization of extracellular proteins into supramolecular structure. ${ }^{17-19}$ The results of these 
findings suggest that each missense mutation may lead to the lack of assembly of secretion, and may reduce the incorporation of $\alpha$-tectorin into the tectorial membrane.

In this study, we have reported the prevalence of TECTA mutations in Japanese ADNSHL patients detected by genetic screening, and confirmed the genotype-phenotype correlations. We also elucidated how mutation in the ZP domain of $\alpha$-tectorin causes hearing loss through protein expression study of ZP domain proteins. TECTA mutation screening should be considered for patients with mild to moderate inherited $\mathrm{AD}$ hearing loss because of its higher incidence. Further investigation of this gene is necessary to identify the function in the cochlea responsible for the distinct phenotype.

\section{ACKNOWLEDGEMENTS}

We sincerely thank the families for their participation in this study. And we also thank AC Apple-Mathews for help in preparing the manuscript. This work was supported by the Ministry of Health and Welfare, Japan (SU), and a grantin-aid for Scientific Research from the Ministry of Education, Science and Culture of Japan (SU).

1 Smith, R. J., Bale, Jr J. F. \& White, K. R. Sensorineural hearing loss in children. Lancet 365, 879-890 (2005).

2 Petersen, M. B. \& Willems, P. J. Non-syndromic, autosomal-recessive deafness. Clin. Genet. 69, 371-392 (2006).

3 Verhoeven, K., Van Laer, L., Kirschhofer, K., Legan, P. K., Hughes, D. C., Schatteman, I. et al. Mutations in the human alpha-tectorin gene cause autosomal dominant nonsyndromic hearing impairment. Nat. Genet. 19, 60-62 (1998).

4 Moreno-Pelayo, M. A., del Castillo, I., Villamar, M., Romero, L., Hernandez-Calvin, F. J., Herraiz, C. et al. A cysteine substitution in the zona pellucida domain of alphatectorin results in autosomal dominant, postlingual, progressive, mid frequency hearing loss in a Spanish family. J. Med. Genet. 38, E13 (2001).

5 Iwasaki, S., Harada, D., Usami, S., Nagura, M., Takeshita, T. \& Hoshino, T. Association of clinical features with mutation of TECTA in a family with autosomal dominant hearing loss. Arch. Otolaryngol. Head Neck Surg. 128, 913-917 (2002).
6 Plantinga, R. F., de Brouwer, A. P., Huygen, P. L., Kunst, H. P., Kremer, H. \& Cremers, C. W. A novel TECTA mutation in a Dutch DFNA8/12 family confirms genotypephenotype correlation. J. Assoc. Res. Otolaryngol. 7, 173-181 (2006).

7 Sagong, B., Park, R., Kim, Y. H., Lee, K. Y., Baek, J. I., Cho, H. J. et al. Two nove missense mutations in the TECTA gene in Korean families with autosomal dominant nonsyndromic hearing loss. Ann. Clin. Lab. Sci. 40, 380-385 (2010).

8 Hildebrand, M. S., Morin, M., Meyer, N. C., Mayo, F., Modamio-Hoybjor, S. Mencia, A. et al. DFNA8/12 caused by TECTA mutations is the most identified subtype of nonsyndromic autosomal dominant hearing loss. Hum. Mutat. 32, 825-834 (2011).

9 de Heer, A. R., Pauw, R. J., Huygen, P. L., Collin, R. W., Kremer, H. \& Cremers, C. W. Flat threshold and mid-frequency hearing impairment in a Dutch DFNA8/12 family with a novel mutation in TECTA. Some evidence for protection of the inner ear. Audiol. Neurootol. 14, 153-162 (2009).

10 Balciuniene, J., Dahl, N., Jalonen, P., Verhoeven, K., Van Camp, G., Borg, E. et al. Alpha-tectorin involvement in hearing disabilities: one gene-two phenotypes. Hum. Genet. 105, 211-216 (1999).

11 Legan, P. K., Lukashkina, V. A., Goodyear, R. J., Kossi, M., Russell, I. J. \& Richardson, G. P. A targeted deletion in alpha-tectorin reveals that the tectorial membrane is required for the gain and timing of cochlear feedback. Neuron 28, 273-285 (2000).

12 Plantinga, R. F., Cremers, C. W., Huygen, P. L., Kunst, H. P. \& Bosman, A. J. Audiological evaluation of affected members from a Dutch DFNA8/12 (TECTA) family. J. Assoc. Res. Otolaryngol. 8, 1-7 (2007).

13 Sarkar, G. \& Sommer, S. S. The 'megaprimer' method of site-directed mutagenesis. Biotechniques 8, 404-407 (1990).

14 Govaerts, P. J., De Ceulaer, G., Daemers, K., Verhoeven, K., Van Camp, G., Schatteman, I. et al. A new autosomal-dominant locus (DFNA12) is responsible for a nonsyndromic, midfrequency, prelingual and nonprogressive sensorineural hearing loss. Am. J. Otol. 19, 718-723 (1998).

15 Moreno-Pelayo, M. A., Goodyear, R. J., Mencia, A., Modamio-Hoybjor, S., Legan, P. K., Olavarrieta, L. et al. Characterization of a spontaneous, recessive, missense mutation arising in the Tecta gene. J. Assoc. Res. Otolaryngol. 9, 202-214 (2008).

16 Pfister, M., Thiele, H., Van Camp, G., Fransen, E., Apaydin, F., Aydin, O. et al. A genotype-phenotype correlation with gender-effect for hearing impairment caused by TECTA mutations. Cell Physiol. Biochem. 14, 369-376 (2004).

17 Jovine, L., Qi, H., Williams, Z., Litscher, E. \& Wassarman, P. M. The ZP domain is a conserved module for polymerization of extracellular proteins. Nat. Cell. Biol. 4, 457-461 (2002).

18 Qi, H., Williams, Z. \& Wassarman, P. M. Secretion and assembly of zona pellucida glycoproteins by growing mouse oocytes microinjected with epitope-tagged cDNAs for mZP2 and mZP3. Mol. Biol. Cell 13, 530-541 (2002).

19 Jovine, L., Darie, C. C., Litscher, E. S. \& Wassarman, P. M. Zona pellucida domain proteins. Ann. Rev. Biochem. 74, 83-114 (2005). 